\title{
Combined treatment with metformin and gefitinib overcomes primary resistance to EGFR-TKIs with EGFR mutation via targeting IGF-IR signaling pathway
}

This article was published in the following Dove Press journal:

Biologics:Targets and Therapy

\author{
Yong-hong Pan \\ Lin Jiao \\ Cai-yu Lin \\ Cong-hua Lu \\ $\mathrm{Li} \mathrm{Li}$ \\ Heng-yi Chen \\ Yu-bo Wang \\ Yong $\mathrm{He}$
}

Department of Respiratory Disease, Daping Hospital, Third Military

Medical University, Chongqing 400042, China
Correspondence: Yong $\mathrm{He}$ Department of Respiratory Disease, Daping Hospital, Third Military Medical University, No 10 Changjiang Zhi Road, Yuzhong District, Chongqing 400042, China

Tel/fax +862368757791

Email heyong 8998@126.com

\begin{abstract}
Aim: Although EGFR tyrosine kinase inhibitors (TKIs) have shown dramatic effects against sensitizing EGFR mutations in non-small cell lung cancer (NSCLC), $20 \%-30 \%$ of NSCLC patients with EGFR-sensitive mutation exhibit intrinsic resistance to EGFR-TKIs. The purpose of the current study was to investigate the enhanced antitumor effect of metformin (Met), a biguanide drug, in combination with gefitinib (Gef) in primary resistant human lung cancer cells and the associated molecular mechanism.
\end{abstract}

Experimental design: H1975 cell line was treated with Met and/or Gef to examine the inhibition of cell growth and potential mechanism of action by using 3-(4,5-dimethylthiazol-2-yl)2,5-diphenyltetrazolium bromide (MTT), Ki67 incorporation assay, flow cytometry analysis, small interfering RNA technology, Western blot analysis and xenograft implantation.

Results: Insulin-like growth factor-1 receptor (IGF-1R) signaling pathway was markedly activated in EGFR-TKI primary resistant $\mathrm{H} 1975$ cells as compared to EGFR-TKI acquired resistance cells (PC-9GR, H1650-M3) and EGFR-TKI sensitivity cells (PC-9, HCC827). Inhibition of IGF-1R activity by AG-1024 (a small molecule of IGF-1R inhibitor), as well as downregulation of IGF-1R by siRNA, significantly enhanced the ability of Gef to suppress proliferation and induce apoptosis in $\mathrm{H} 1975$ cells via the inhibition of AKT activation and subsequent upregulation of Bcl-2-interacting mediator of cell death (BIM). Interestingly, the observation showed that Met combined with Gef treatment had similar tumor growth suppression effects in comparison with the addition of AG-1024 to therapy with Gef. A clear synergistic antiproliferative interaction between Met and Gef was observed with a combination index (CI) value of 0.65 . Notably, IGF-1R silencing mediated by RNA interference (RNAi) attenuated anticancer effects of Met without obviously resensitizing H1975 cells to Gef. Finally, Met-based combinatorial therapy effectively blocked tumor growth in the xenograft with TKI primary resistant lung cancer cells. Conclusion: Our findings demonstrated that Met combined with Gef would be a promising strategy to overcome EGFR-TKI primary resistance via suppressing IGF-1R signaling pathway in NSCLC.

Keywords: metformin, gefitinib, IGF-1R, primary resistance, lung cancer

\section{Introduction}

Non-small cell lung cancer (NSCLC) patients with sensitizing EGFR mutations, such as exon 19 deletions and L858R point mutations, are greatly responsive to the firstgeneration reversible EGFR tyrosine kinase inhibitors (TKIs) of gefitinib (Gef) and erlotinib without serious side effects. ${ }^{1,2}$ Several large Phase III clinical trials have confirmed the efficacy and safety of Gef, which has been recommended as the standard first-line 
treatment for advanced NSCLC patients harboring activating EGFR mutations. ${ }^{3,4}$ However, drug resistance greatly limits the efficacy of the EGFR-TKI treatment. The study of resistance to EGFR-TKIs in EGFR-mutant NSCLC patients can be divided into two broad categories: acquired and primary resistance. Acquired resistance refers to patients who invariably develop progressive disease following an initial objective response or a prolonged stable disease in $\sim 9-13$ months, ${ }^{5}$ whereas primary resistance refers to patients who have progressive disease in $<3$ months or stable disease as the best response to EGFRTKIs. ${ }^{6,7}$ All patients with activating EGFR mutations who are initially responsive to EGFR-TKIs develop acquired resistance inevitably, while $\sim 20 \%-30 \%$ of patients reveal primary resistance to EGFR-TKIs despite having tumors that harbor EGFR mutations. ${ }^{8-11}$ Resistance to EGFR-TKI treatment is a major obstacle in effective treatment of NSCLC. Therefore, our group engaged in identifying an effective, economical and safe combined therapeutic strategy to overcome drug resistance to EGFR-TKIs in NSCLC patients.

Metformin (Met) is a commonly used oral antihyperglycemic agent for type 2 diabetes mellitus (T2DM), displaying significant antitumor properties in various types of cancers including breast, prostate and ovary. ${ }^{12-14}$ In the T2DM patients, Met treatment is associated with decreased incidence and mortality of cancer in comparison with other antidiabetic agents. ${ }^{15}$ A retrospective observational trial from our group demonstrated that Met, in contrast with other hypoglycemic agents, combined with EGFR-TKI treatment was significantly associated with survival benefit outcome of advanced NSCLC patients with diabetes. ${ }^{16}$ Previously, our group has confirmed that Met co-treatment with EGFR-TKIs could overcome acquired resistance, leading to strong inhibition on acquired resistance of NSCLC cells' proliferation, migration, and invasion synergistically. ${ }^{17}$ However, it remains unknown whether Met may sensitize primary resistant NSCLC to EGFR-TKIs.

The molecular mechanisms associated with acquired resistance to EGFR-TKIs have been well documented. ${ }^{18-22}$ On the other hand, there are only a few preclinical and retrospective reports on the mechanism of the primary resistance to EGFR-TKIs. It has been reported that de novo MET amplification, ${ }^{23,24} \mathrm{Bcl}-2$ interacting mediator of cell death (BIM)-deletion polymorphism, ${ }^{25}$ phosphatase and tensin homolog (PTEN) $\operatorname{loss}^{26}$ and de novo T790M mutation ${ }^{27,28}$ are associated with intrinsic resistance to EGFR-TKIs, but still a lot of primary resistance mechanisms are largely unknown. The insulin-like growth factor-1 receptor (IGF-1R) signaling activation has recently been reported to play a key role in primary resistance of EGFR-TKIs. ${ }^{29}$ Having IGF1-positive serum indicated a negative prognosis in EGFR activating mutation in NSCLC patients who received Gef therapy. ${ }^{30}$ EGFR-mutant NSCLC patients with de novo EGFR-TKI resistance showed a high expression of IGF-1R. ${ }^{31}$

Here, our study demonstrated that compared to EGFRTKI-sensitive cells (HCC827 and PC-9) and EGFR-TKI acquired resistant cells (PC-9GR and H1650-M3), the IGF$1 \mathrm{R}$ activity was much higher in EGFR-TKI primary resistant H1975 cells. However, primary resistance was attenuated upon incubation with the Gef and IGF-1R pathway inhibitor AG-1024 or when IGF-1R expression was reduced through RNA interference (RNAi). This treatment displayed reduction in phosphorylation of Akt kinase, the major downstream regulator of IGF-1R signaling, as well as upregulation of BIM and increased apoptosis potential in vitro. Met effectively increased the sensitivity of primary resistant H1975 cells to Gef, resulting in growth inhibition and apoptosis induction, via inhibiting IGF-1R signaling by regulating the AKT/BIM pathway. Furthermore, knockdown of IGF-1R expression incubation with siRNA abolished the synergistic effect of Met on Gef-induced cytotoxicity in H1975 cells. In addition, this combinational therapy reduced tumor masses much more effectively than Gef alone in a xenograft mouse model.

\section{Materials and methods Cell culture and reagents}

EGFR-TKI-sensitive PC-9 cells were gifted by Prof. J. Xu and Dr. M. Liu from Guangzhou Medical University (China), and HCC827 cells were provided by the American Type Culture Collection. EGFR-TKI acquired resistant PC-9GR cells were gifted by Prof. J. Xu and Dr. M. Liu from Guangzhou Medical University (China), and H1650-M3 cells were kindly provided by Dr. R. Sordella from Cold Spring Harbor Laboratory. EGFR-TKI primary resistant H1975 cells were provided by the American Type Culture Collection. All the cells were cultured in the Roswell Park Memorial Institute 1640 medium (RPMI-1640; HyClone; HyClone, Logan, UT, USA) with Earle's salts and supplemented with $10 \%$ fetal bovine serum (FBS; Thermo Fisher Scientific, Waltham, MA, USA), 2 mM L-glutamine solution (Thermo Fisher Scientific), $100 \mathrm{U} / \mathrm{mL}$ penicillin (HyClone) and $100 \mu \mathrm{g} / \mathrm{mL}$ streptomycin sulfate (HyClone) at $37^{\circ} \mathrm{C}$ with $5 \% \mathrm{CO}_{2}$ in the air and 90\% humidity. Gef (Iressa), purchased from Tocris Bioscience (Ellisville, MO, USA), and AG-1024, purchased from Selleck Chemicals (Houston, TX, USA), were prepared in dimethyl sulfoxide (DMSO) to obtain a stock solution of $10 \mathrm{mmol} / \mathrm{L}$. Met (Sigma-Aldrich Co., St Louis, MO, USA) was dissolved in deionized water, and a $1 \mathrm{M}$ stock solution 
was prepared and stored in aliquots at $-20^{\circ} \mathrm{C}$. The use of gifted cell lines was approved by ethics committee of the Third Military Medical University.

\section{3-(4,5-dimethylthiazol-2-yl)-2,5- diphenyltetrazolium bromide (MTT) assay} Cytotoxicity of Gef plus Met was evaluated by the MTT dye reduction assay. A total of 2000 cells were plated in a $100 \mu \mathrm{L}$ culture medium in 96-well microtiter plates. After $12 \mathrm{~h}$ incubation, Gef and $5 \mathrm{mM}$ Met were added to each well as indicated, and the cells were further cultured for $48 \mathrm{~h}$. Then, $10 \mu \mathrm{L}$ of $5 \mathrm{mg} / \mathrm{mL}$ MTT reagent (Sigma-Aldrich Co.) in a $200 \mu \mathrm{L}$ culture medium was added to each well. After $4 \mathrm{~h}$, the medium was removed, and $150 \mu \mathrm{L}$ of DMSO was admixed to each well to dissolve the formazan crystals. Absorbance was measured at a wavelength of $490 \mathrm{~nm}$ by using a ThermoFisher Spectrophotometer1510 (Molecular Devices LLC, Sunnyvale, CA, USA). Cell viability was determined by dividing the absorbance values of the treated cells with that of the untreated cells. Cytotoxic activity was recorded as $\mathrm{IC}_{50}$, which is the concentration necessary to reduce the absorbance of treated cells by $50 \%$ compared to the control (untreated cells). The experiments were conducted in triplicates.

\section{Ki67 incorporation assay}

Cell proliferation was also assessed by the Ki67 incorporation assay using a Ki67 labeling and detection kit (SigmaAldrich Co.). Briefly, cells were treated with Met or Gef, or both, for $48 \mathrm{~h}$ and then were incubated for $12 \mathrm{~h}$ with Ki67 (1:200 dilution) and fixed. Cell nuclei were counterstained with 4',6-diamidino-2-phenylindole (DAPI) and then viewed with a live cell station (API DeltaVision, Rockville, MD, USA). At least 500 cells from three independent experiments were counted. The data obtained were expressed as the mean value of the percentage of positive cells \pm standard error of the mean (SEM).

\section{Calculation of combination index $(\mathrm{Cl})$ and data analysis}

The isobolographic method was used to measure the type of interaction between Met and Gef. The CI was calculated for combinations of Met and Gef against H1975 cells, and the results were interpreted as described in the following: ${ }^{32}$

$\mathrm{CI}=(D) 1 /(D x) 1+(D) 2 /(D x) 2+a(D) 1(D) 2 /(D x) 1(D x) 2$

where $(D x) 1=\mathrm{IC}_{50}$ of drug 1 (Met) alone; $(D) 1=\mathrm{IC}_{50}$ of drug 1 (Met) in combination with drug 2 (Gef); $(D x) 2=\mathrm{IC}_{50}$ of drug $2(\mathrm{Gef})$ alone; $(D) 2=\mathrm{IC}_{50}$ of drug $2(\mathrm{Gef})$ in combination with drug 1 (Met) and $\alpha=0$ for mutually exclusive or $\alpha=1$ for mutually nonexclusive modes of drug action.

\section{Interpretation of results}

Antagonism for CI value of $>1.3$, moderate antagonism for CI value of 1.1-1.3, additive interaction for CI value of $0.9-1.1$, slight synergism for CI value of $0.8-0.9$, moderate synergism for CI value of $0.4-0.6$, and strong synergism for CI value of $0.2-0.4$ were found.

\section{Apoptosis assay}

Flow cytometric analysis was used to detect apoptosis by examining altered plasma membrane phospholipid packing by the lipophilic dye Annexin V. Briefly, cells were treated with Gef and/or Met for $48 \mathrm{~h}$, harvested by trypsin, washed twice with PBS and then resuspended at a density of $1 \times$ $10^{7}$ cells $/ \mathrm{mL}$. Thereafter, $5 \mu \mathrm{L}$ of Annexin V-FITC and $5 \mu \mathrm{L}$ of propidium iodide (PI) were added to $100 \mu \mathrm{L}$ of the cell suspension and incubated for $30 \mathrm{~min}$ at room temperature in the dark. Next, labeled cells were processed by flow cytometry. All early apoptotic cells (i.e., Annexin V positive, PI negative), necrotic/late apoptotic cells (i.e., double positive) and living cells (i.e., double negative) were detected by using a Cytomics FC 500 flow cytometer (Beckman Coulter, Miami, FL, USA).

\section{Transfection of siRNAs}

H1975 cells were seeded at 40\% cell confluency per well in six-well plates.

IGF-1R-specific siRNA used for IGF-1R knockdown and the control siRNA were synthesized by GenePharma (Shanghai GenePharma Co., Ltd.). The sequences of siRNA targeting the IGF-1R coding region were as follows: sense, 5'-GGAGAGAACUGUCAUUUCUTT-3' and antisense, 5'-AGAAAUGACAGUUCUCUCCTT-3'. The siRNAs were then transfected into the $\mathrm{H} 1975$ cells by HiPerFect Transfection Reagent (Qiagen, Hilden, Germany) according to the manufacturer's instructions.

\section{Western blot assay}

Cells grown and treated as indicated were collected, and the total protein was extracted. The following primary antibodies were purchased from Cell Signaling Technology, Inc. (CST; Cambridge, MA, USA) and used: rabbit monoclonal anti-phosphorylated IGF-1R (Tyr1131), rabbit monoclonal anti-IGF-1R, rabbit monoclonal anti-AKT, rabbit monoclonal anti-phosphorylated AKT (Ser473), and rabbit monoclonal anti-Bim. Horseradish peroxidase-conjugated 
goat-anti-rabbit antibody (Thermo Fisher Scientific) was used as a secondary antibody. The control for equal protein loading was assessed using an anti-GAPDH antibody (CST).

\section{Animal experiments}

For xenograft implantation, a total of $2 \times 10^{6}$ H1975 cells were injected subcutaneously into the back, next to the left forelimb of 6-week-old female BALB/cA-nu mice (Laboratory Animal Center of Third Military Medical University, Chongqing, China), all of which developed tumors with a size of $30 \mathrm{~mm}^{3}$ within 5-7 days. The mice were then randomly assigned to two groups (eight mice/group) with or without oral administration of $1 \mathrm{mg} / \mathrm{mL}$ Met and $250 \mathrm{mg} / \mathrm{L}$ Gef in drinking water. Tumor volume was calculated as (length $\times$ width $^{2}$ )/ 2 and measured twice a week. The animals were kept in individual ventilated cages in compliance with institutional guidelines. After 4 weeks, tumor-bearing mice were euthanized by intraperitoneal overdose of sodium pentobarbital and tumors were harvested and weighed at the end. All animal protocols were approved by the ethics committee of the Third Military Medical University with the approval number of SYXK(YU)20170002 and were performed in accordance with the guidelines of the Animal Welfare Act of the National Institutes of Health (NIH publication no 80-23), revised 2010.

\section{Statistical analysis}

All data are presented as mean \pm SD. Statistical analysis among the groups was analyzed by one-way analysis of variance (ANOVA) with a post hoc Bonferroni adjustment using Statistical Package for the Social Sciences (SPSS) software (version 21.0; SPSS Inc., Chicago, IL, USA), and statistical significance was assumed at an alpha value of $p<0.05$.

\section{Results}

\section{Primary resistance to EGFR-TKIs was more dependent on IGF-IR signaling pathway}

We first detected the basal expression of p-IGF-1R, IGF-1R in a series of NSCLC cells. As shown in Figure 1, although no obvious change was detected in the basal levels of total IGF-1R, EGFR-TKIs primary resistant H1975 cells exhibited higher levels of p-IGF-1R than acquired resistant cells (PC9GR, H1650-M3) and sensitized cells (HCC827, PC-9). Our results demonstrated that primary resistance to EGFR-TKIs was more dependent on the IGF-1R pathway (Figure 1).

\section{IGF-IR activation was responsible for EGFR-TKI primary resistance}

To clearly identify whether IGF-1R signals contribute to the EGFR-TKI primary resistance, we used a small molecule chemical inhibitor of IGF-1R (AG-1024) against IGF-1R activity in H1975 cells. Western blot analysis showed that AG-1024 treatment indicated a specific reduction in phosphorylation protein levels of IGF-1R compared to control. When it was combined with Gef, IGF-1R activity was markedly suppressed despite the total-IGF-1R protein not being changed (Figure 2D).

Next, we further confirmed the effect of IGF-1R activity inhibitor using AG-1024 on H1975 cell proliferation. MTT assay was conducted to examine the growth inhibitory effect of AG-1024, Gef and combinational therapy on H1975 cells at $48 \mathrm{~h}$ of treatment. Results showed that AG-1024 induced a dose- and time-dependent decrease in cell viability of $\mathrm{H} 1975$ cells with $\mathrm{IC}_{50}$ values of $43.6 \pm 2.4 \mu \mathrm{M}$. Meanwhile, the indicated concentration of Gef alone determined a cytotoxic effect
H1975 HCC827 PC-9 PC-9GR H1650-M3

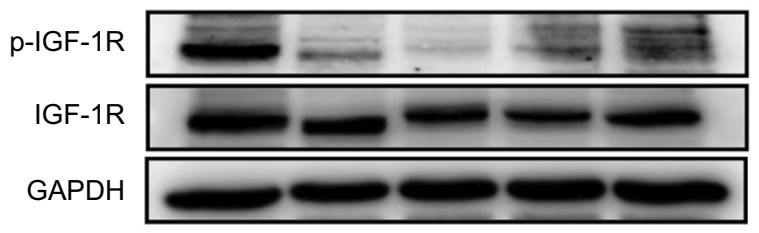

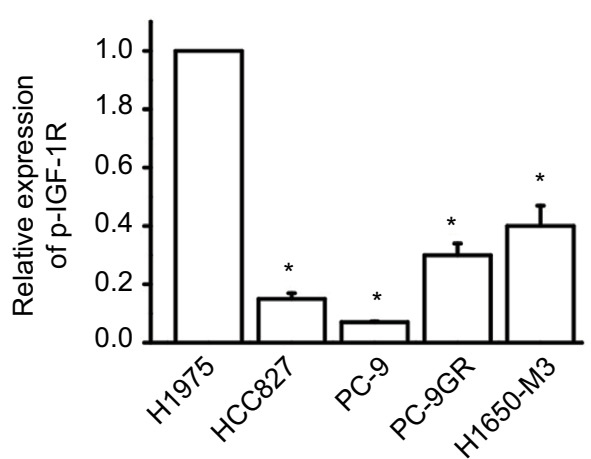

Figure I High IGF-IR activation in HI 975 cells

Notes: TKI primary resistant HI975 cells, TKI acquired resistant PC-9GR and HI650-M3 cells, TKI-sensitized HCC827 and PC-9 cells were incubated in the $10 \%$ FBS medium. Equal amounts of whole cell lysates $(30 \mu \mathrm{g})$ were subjected to electrophoresis, and the basal expressions of IGF-IR and p-IGF-IR were analyzed by Western blot for indicated antibodies. Relative expression of $p-I G F-I R$ was expressed as mean \pm SD from three independent experiments. ${ }^{*} p<0.00 I$ (one-way ANOVA).

Abbreviations: IGF-IR, insulin-like growth factor-I receptor; TKI, tyrosine kinase inhibitor; FBS, fetal bovine serum; ANOVA, analysis of variance. 
on $\mathrm{H} 1975$ cells with $\mathrm{IC}_{50}$ values of $9.4 \pm 0.74 \mu \mathrm{M}$; when it was combined with $20 \mu \mathrm{M}$ of AG-1024, it showed a significant synergistic antiproliferative effect on $\mathrm{H} 1975$ cells with its $\mathrm{IC}_{50}$ values dropping to $2.8 \pm 0.2 \mu \mathrm{M}$ ( $p=0.00$; Figure $2 \mathrm{~A}$ and $\mathrm{B})$.

We also assessed the synergistic effect of IGF-1R inhibitor treatment in combination with Gef in $\mathrm{H} 1975$ cells through Ki67 incorporation assay. Ki67 labeling (as a percentage of total nuclei) was obviously reduced under combination treatment of AG-1024 and Gef (Figure 2C).

To investigate the possible mechanism by which IGF1R signaling was inhibited on H1975 cells, the activities of IGF-1R downstream molecules were examined. Results showed the effects of single treatment with AG-1024 on inhibition of AKT activities and elevation of BIM protein level were modest, whereas the combination of AG-1024 and Gef substantially suppressed AKT activities and further induced apoptotic proteins of BIM (Figure 2D). These results demonstrated that co-treatment with AG-1024 and Gef could synergistically induce H1975 cells apoptosis by controlling AKT/BIM pathways.

These data indicated that IGF-1R activation was responsible for the proliferation of EGFR-TKI primary resistance
A

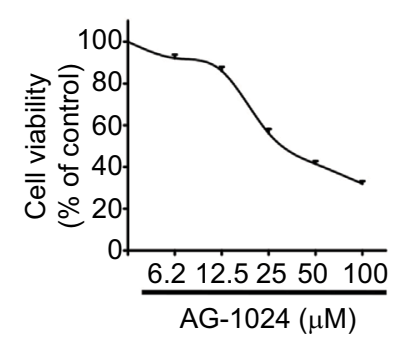

C

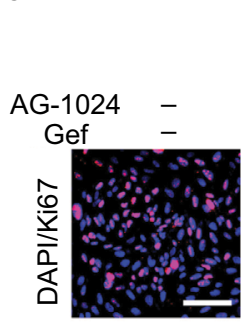

D

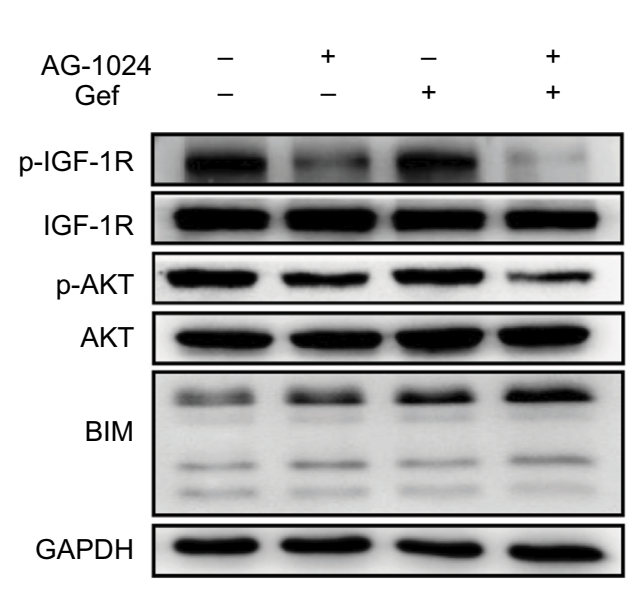

B
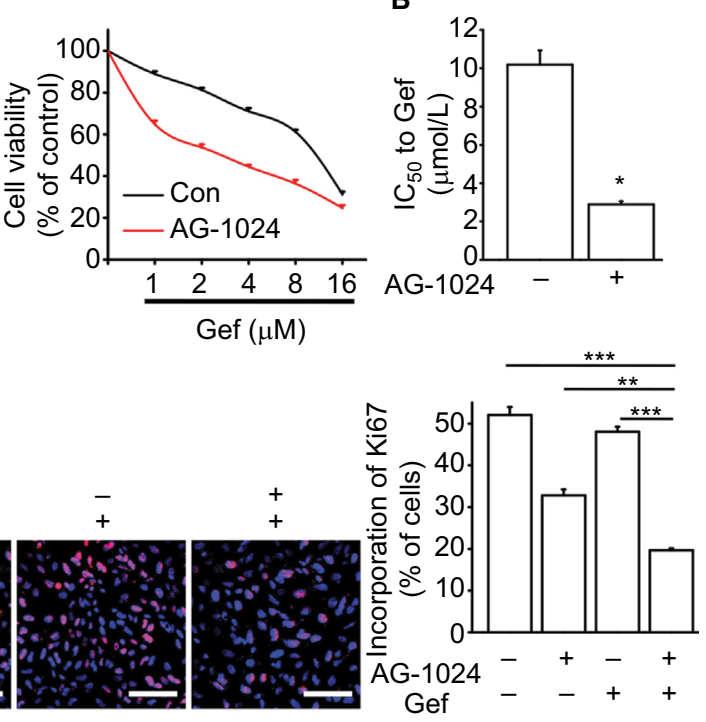

Gef

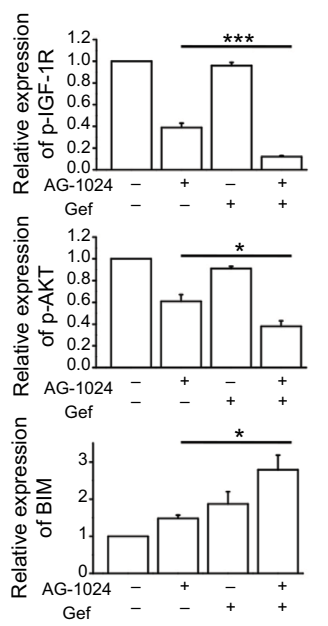

Figure 2 IGF-IR inhibitor enhanced Gef cytotoxicity in HI 975 cells.

Notes: (A) Cells were treated with indicated doses of AG-1024 or Gef or a combination of AG-1024 (20 $\mu$ M) with Gef for 48 h. Cell viability was measured by MTT assay. (B) IC $C_{50}$ values of Gef toward HI975 cells for $48 \mathrm{~h}$ with or without AG-1024 treatment, ${ }^{*} p<0.05$. (C) AG-1024 (20 $\mu$ M) combined with Gef $(4 \mu M)$ synergistically inhibited the proliferation of $\mathrm{HI} 975$ cells, as determined by a Ki67 incorporation assay. Statistical differences were calculated by one-way ANOVA. $* * p<0.0 \mathrm{I}$ and $* * * p<0.00 \mathrm{I}$. Scale bars: $80 \mu \mathrm{m}$. (D) Cells were treated with AG-1024 (20 $\mu \mathrm{M})$ and/or Gef $(4 \mu \mathrm{M})$; after $48 \mathrm{~h}$, cells were harvested and subjected to Western blot using the indicated antibodies. Relative expression values of $p-I G F-I R, p-A K T$ and BIM were expressed as mean \pm SD from three independent experiments. * $p<0.05$ and $* * * p<0.00$ I (one-way ANOVA). Abbreviations: IGF-IR, insulin-like growth factor-I receptor; Gef, gefitinib; MTT, 3-(4,5-dimethylthiazol-2-yl)-2,5-diphenyltetrazolium bromide; ANOVA, analysis of variance; BIM, Bcl-2-interacting mediator of cell death; DAPI, 4',6-diamidino-2-phenylindole. 
in NSCLC; inhibiting IGF-1R activity could overcome EGFR-TKI primary resistance by downregulation of AKT phosphorylation and upregulation of BIM.

\section{Met sensitized HI975 cells to Gef}

To evaluate the antiproliferative effect of Met on H1975 cells, we first performed MTT assays and found that Met could enhance the inhibitory action of Gef on the growth of $\mathrm{H} 1975$ cells, with $\mathrm{IC}_{50}$ values varying from $9.41 \pm 0.96 \mu \mathrm{M}$ to $2.06 \pm 0.60 \mu \mathrm{M}$ (Figure 3A and B).

Considering Met could disrupt mitochondrial respiration, which may affect the MTT assay results, we further performed Ki67 incorporation assay and found that Met exhibited a profound synergistic inhibition effect on H1975 cells when it was combined with Gef (Figure 3C).
Next, we performed flow cytometry analysis to determine whether the drug combination could enhance apoptosis. Flow cytometric analysis demonstrated that few Met-treated cells or Gef-treated cells underwent apoptosis. However, combination therapy of Met with Gef markedly enhanced the apoptotic cells (Figure 3D). These results suggest that Met could sensitize H1975 cells to Gef by reducing cell viability and inducing cell apoptosis.

\section{Cytotoxic effect of Met and/or Gef on HI 975 cells}

A dose-dependent inhibition of $\mathrm{H} 1975$ cells proliferation was observed after treatment with Met or Gef with $\mathrm{IC}_{50}$ values of $21.44 \mathrm{mM}$ or $9.41 \mu \mathrm{M}$, respectively (Table 1). Testing different combinations of Met and Gef showed a clear synergistic
A
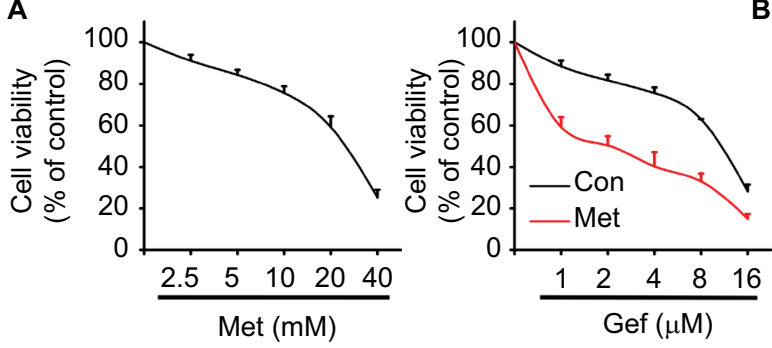

B

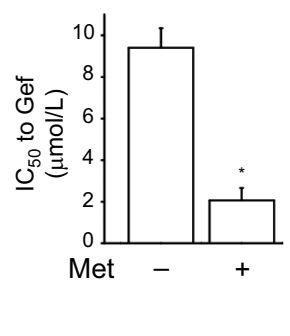

C

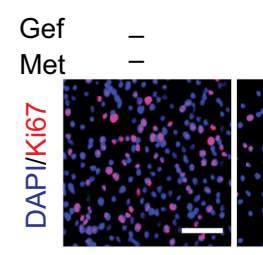

$\overline{+}$

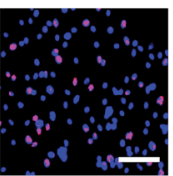

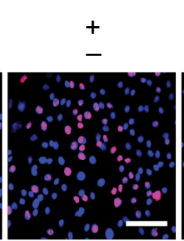
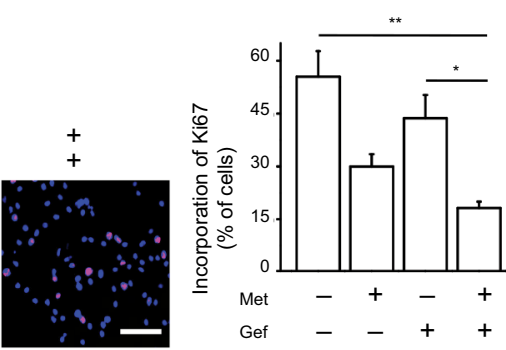
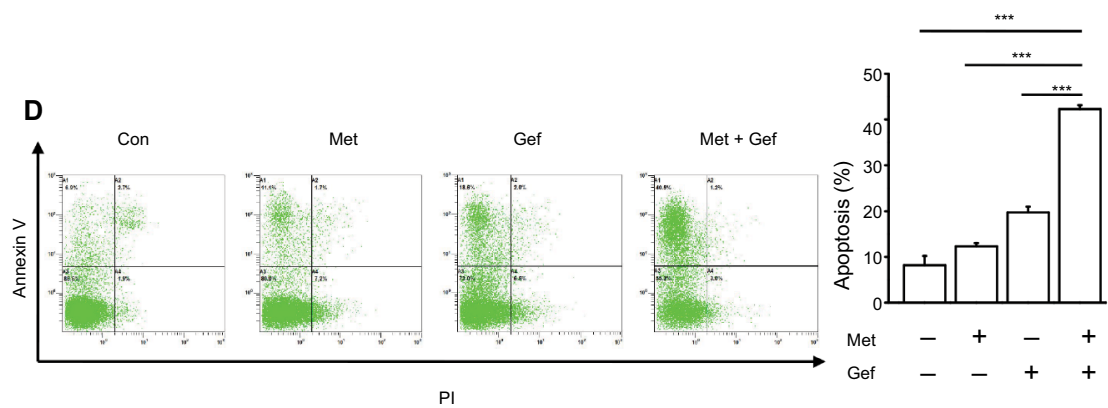

Figure 3 Met sensitized HI975 cells to Gef.

Notes: (A) Cells were treated with different concentrations of Met and Gef in the absence or presence of Met (5 mM) for $48 \mathrm{~h}$, and viability of cells was then measured using the MTT assay. (B) IC 50 values of HI 975 cells to Gef; ${ }^{*} p<0.01$ compared with that without Met treatment. (C) The synergistic antiproliferation effects of Met (5 mM) combined with Gef $(4 \mu \mathrm{M})$ on HI975 cells, as determined by a Ki67 incorporation assay. Statistical differences between groups were analyzed using one-way ANOVA.

${ }^{*} p<0.05$ and ${ }^{* *} p<0.01$. (D) Cells were incubated as $(\mathbf{C})$, and apoptosis was assessed by Annexin V/PI staining and FACS analysis. *** $p<0.00 \mathrm{I}$. The images are representative of three independent experiments.

Abbreviations: Met, metformin; Gef, gefitinib; MTT, 3-(4,5-dimethylthiazol-2-yl)-2,5-diphenyltetrazolium bromide; ANOVA, analysis of variance; PI, propidium iodide; FACS, fluorescence-activated cell sorting; DAPI, 4',6-diamidino-2-phenylindole; Con, control. 
interaction $(\mathrm{CI}=0.65)$ with reduction in the $\mathrm{IC}_{50}$ values for both agents to $7.42 \mathrm{mM}$ and $2.06 \mu \mathrm{M}$, respectively (Table 1).

\section{Met decreased IGF-IR signaling activation in HI 975 cells}

IGF-1R signaling plays a key role in regulating the growth of H1975 cells, and IGF-1R inhibitor suppresses growth and induces apoptosis on H1975 cells. In addition, the combination of Met and Gef leads to inhibition of H1975 cells' proliferation and induction of apoptosis. To explore the mechanism underlying the antitumor effects of Met on H1975 cells, the effects of Met on IGF-1R signaling pathway were detected. We performed Western blot assay and found that Met alone inhibited IGF-1R activation and regulated its downstream signaling proteins such as suppressing $\mathrm{p}-\mathrm{AKT}$ or escalating BIM, and Gef alone produced no significant change in the IGF-1R signaling pathway. After Met was combined with Gef, a more pronounced decrease in the protein levels of p-IGF-1R and p-AKT and increase in the protein levels of BIM were seen. These results show that Met promoted apoptosis of H1975 cells via regulating the IGF-1R/AKT/ BIM signaling pathway (Figure 4).

\section{Met resensitized primary resistant HI975 cells to Gef highly dependent on IGF-IR signaling inhibition}

To verify whether Met resensitizes EGFR-TKIs innate resistance cells to Gef through inhibiting IGF-1R signaling, IGF-1R siRNA was transfected into H1975 cells to downregulate IGF-1R expression. Western blot analysis showed a marked reduction of IGF-1R expression in H1975 cells transfected with si-IGF-1R RNA compared with those transfected with siRNA negative control (NC; Figure 5A). As a result, downregulation of IGF-1R decreased p-AKT and increased protein of BIM. MTT assay analysis demonstrated that IGF-1R knockdown could partially recover sensitivity of H1975 cells to Gef in comparison with NC. In addition, co-treatment with Met and Gef inhibited the proliferation of IGF-1R knockdown cells and NC cells to a similar extent. There was no significant difference in Gef sensitivity of

Table I The $\mathrm{IC}_{50}$ values and $\mathrm{Cl}$ for Met and Gef against $\mathrm{HI} 975$ cell line

\begin{tabular}{|c|c|c|c|c|c|}
\hline Gef IC $_{50}$ & Met IC 50 & Gef $I C_{50}$ in combination & Met $\mathrm{IC}_{50}$ in combination & $\mathbf{C l}$ & Interpretation \\
\hline $9.41 \pm 0.96$ & $21.44 \pm 2.23$ & $2.06 \pm 0.60$ & $7.42 \pm 0.32$ & 0.65 & Synergism \\
\hline
\end{tabular}

Abbreviations: $\mathrm{Cl}$, combination index; Met, metformin; Gef, gefitinib.
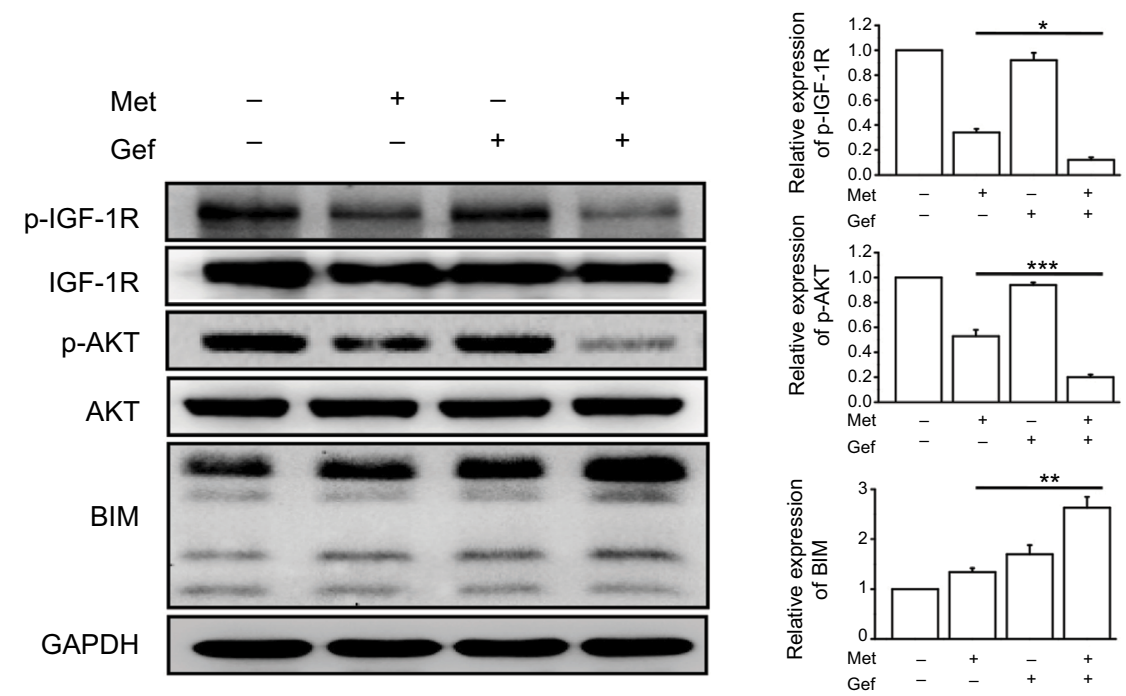

Figure 4 Co-administration of Met and Gef synergically inhibited the activity of IGF-IR signaling pathway in HI975 cells.

Notes: Cells were treated with Met $(5 \mathrm{mM})$ and Gef $(4 \mu \mathrm{M})$ alone or in combination for $48 \mathrm{~h}$, whole cell protein was lysed and immunoblotted with antibodies as indicated, and $\beta$-actin was used to confirm the equal gel loading. Relative expression values of $p$-IGF-IR, $p$-AKT and BIM were expressed as mean \pm SD from three independent experiments. ${ }^{*} p<0.05,{ }^{* *} p<0.01$ and ${ }^{* * *} p<0.001$ (one-way ANOVA).

Abbreviations: Met, metformin; Gef, gefitinib; IGF-IR, insulin-like growth factor-I receptor; BIM, Bcl-2-interacting mediator of cell death; ANOVA, analysis of variance. 
IGF-1R knockdown cells in the presence or absence of Met (Figure 5B and C). These results indicate that Met increased the sensitivity of primary resistant cells to Gef by downregulating mainly the IGF-1R pathway.

\section{Combinational regimen synergistically inhibited tumor growth of HI975 cell xenograft}

Given the synergistic inhibitory effects of Met and Gef on H1975 cells' growth in vitro, it is believed that the combinational regimen has the potential to be highly effective in treating H1975 cells in vivo. Thus, we sought to determine the inhibition effect of Met and Gef on the tumor growth of H1975 cells xenograft. After 24 days, the xenograft tumors of the Gef group grew to an average volume of $2.19 \pm$
$0.29 \mathrm{~cm}^{3}$ starting from $0.05 \pm 0.01 \mathrm{~cm}^{3}$. As shown in Figure $6 \mathrm{~A}$, addition of Met to Gef showed a significant synergistic inhibition of xenograft tumor growth from day $20(p=0.04)$ with an average volume of $0.95 \pm 0.16 \mathrm{~cm}^{3}$. Moreover, gross appearances of xenograft tumors from the combination of Met and Gef, and Gef group are presented in Figure 6C. At the end of experiments, the tumors were isolated and weighed. Compared with the Gef group, the mean tumor weight was significantly less in the combinational group without affecting body weight obviously (Figure 6B).

\section{Discussion}

Primary resistance severely limits the clinical efficacy of first-generation EGFR-TKIs therapy, and understanding the mechanisms of this resistance is pivotal for developing more

A
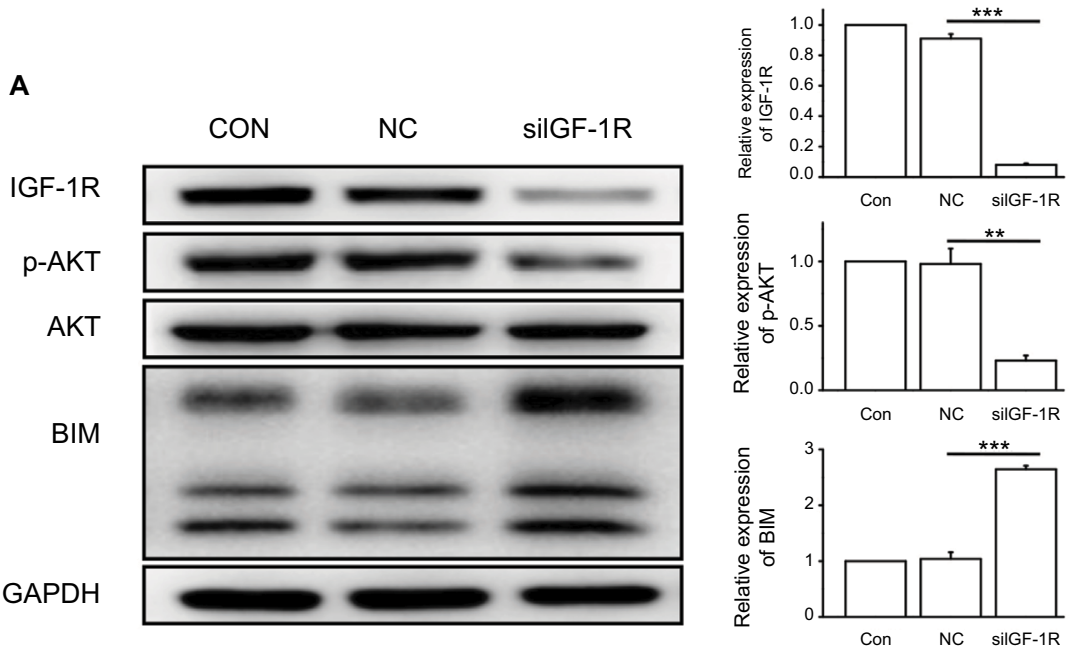

B

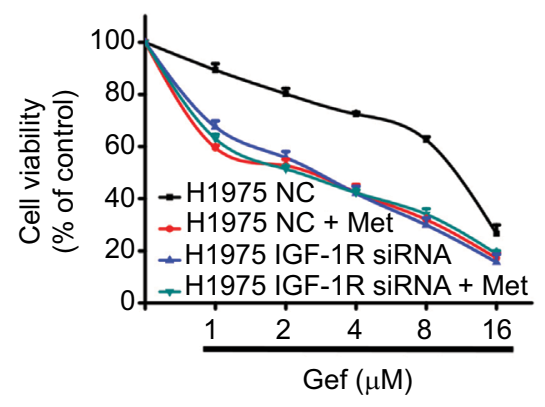

C

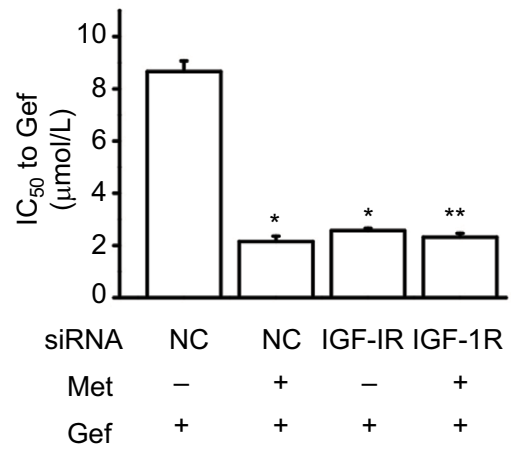

Figure 5 IGF-IR knockdown weakens the anti-proliferation ability of Met in HI975 cells.

Notes: Cells were transfected with IGF-IR siRNA as well as nonspecific siRNA. (A) After transfection, the cells were incubated for $48 \mathrm{~h}$ and the efficiency of silencing was evaluated by Western blot analysis. Relative expression values of IGF-IR, p-AKT and BIM were expressed as mean \pm SD from three independent experiments. $* * p<0.0$ I and ***p $<0.00$ I (one-way ANOVA). (B) After transfection, Met $(5 \mathrm{mM}$ ) only slightly decreased cell viability in HI 975 cells, and the effect of Met ( 5 mM) on Gef sensitivity in HI975 cells was determined by MTT analysis after $48 \mathrm{~h}$ treatment. (C) IC 50 values of Gef toward HI 975 cells after transfection; ${ }^{*} p<0.05$ compared with that of Gef treatment on cells transfected with siRNA NC and **p $>0.05$ compared with that of Gef treatment on cells transfected with siRNA IGF-IR. Images are representative of three independent experiments.

Abbreviations: IGF-IR, insulin-like growth factor-I receptor; Met, metformin; BIM, Bcl-2-interacting mediator of cell death; ANOVA, analysis of variance; MTT, 3-(4,5-dimethylthiazol-2-yl)-2,5-diphenyltetrazolium bromide; Gef, gefitinib; NC, negative control; Con, control. 
effective treatment strategies. Many studies have already reported that activation of the IGF-1R pathway potentially mediates acquired resistance to EGFR-TKIs. ${ }^{33-36}$ Our study provides similar evidence that EGFR-TKI-acquired resistant cell lines of H1650-M3 and PC-9GR exhibited high p-IGF-1R levels, whereas EGFR-TKI-sensitive cell lines of HCC 827 and PC-9 had relatively lower levels of p-IGF-1R. To further examine the role of IGF-1R in primary resistance to EGFR-TKIs, we used the NSCLC H1975 cell line, which reproducibly carries EGFR L858R mutation and de novo EGFR T790M mutation. Our study demonstrated that IGF-1R activity of $\mathrm{H} 1975$ cells was found to be significantly, even higher than that of H1650-M3 and PC-9GR. We also conducted whole-exome sequencing of the H1975 cells; mutation status of Met, PI3KCA and PTEN loss was not observed. In NSCLC H1650 cells with deletion mutation on exon 19, which displays primary resistance to EGFR-TKIs due to exhibiting PTEN loss, ${ }^{26}$ co-treatment of IGF-1R inhibitors such as $\alpha$-IR3, AG-1024 or R1507 with Gef enhanced TKIinduced cells' growth inhibition and apoptosis. ${ }^{37}$ Surprisingly, we found that IGF-1R inhibitor of AG-1024 sensitized H1975 cells, a primary resistance cell line with T790M mutation, to Gef by inducing apoptosis and inhibiting proliferation. Otherwise, knockdown of IGF-1R expression through siRNA could also enhance Gef sensitivity in H1975 cells. Hence, we further reinforce the significance of the deactivated IGF-1R signaling pathway that contributed to overcome EGFR-TKI primary resistance.

AKT signaling pathway plays an important role in cells' multiple biological activities, including proliferation and apoptosis. ${ }^{38,39} \mathrm{~A}$ clinical cancer gene test has described genomic alterations in the AKT pathway; increasing the kinase activity of AKT and contributing to cellular transformation may confer primary resistance to Gef. ${ }^{40}$ IGF-1R can activate the EGFR-shared downstream signaling pathways, one of the signals being the PI3 kinase (PI3K)/AKT pathway. ${ }^{41,42}$ In this study, we selected the key marker of AKT in the shared downstream signaling pathways to verify the effect of IGF-1R inhibitor on the primary resistance to EGFR-TKIs in NSCLC. Our result showed that AG-1024 treatment alone or in combination with Gef suppressed IGF-1R inducement of p-AKT in H1975 cells; the dependence of AKT on IGF-1R signaling was further underscored by a knockdown of IGF-1R using siRNA. Thus, p-AKT status appears to be predominantly controlled by IGF-1R activation in H1975 cells. Of the $\mathrm{BH} 3$-only protein, BIM belonging to the $\mathrm{Bcl}-2$ protein family may promote mitochondria releasing cytochrome $\mathrm{c}$, which, in turn, may activate caspases, the key executioners of mitochondria-mediated apoptosis. ${ }^{43,44}$ AKT signaling pathway deactivation may lead to a substantial increase in BIM protein and induce apoptosis procedure consequently. ${ }^{45,46}$ Based on these studies, we found that downregulation of IGF-1R by siRNA induced expression of pro-apoptotic BH3 protein BIM and addition of AG-1024 to Gef increased the level of BIM protein and, thereby, induced mitochondriamediated apoptosis of primary resistant NSCLC H1975 cells.

In this study, we aimed to explore the effect of Met on EGFR-TKI primary resistance and the underlying mechanism. Our results showed that the combination of Met and Gef induced strong inhibition on $\mathrm{H} 1975$ cells' proliferation to a greater extent than either agent alone, and the apoptosis assay confirmed the synergistic inhibitory effect similarly. Combination of both agents caused a reduction in $\mathrm{IC}_{50}$ values with clear synergistic effect. The enhancement of tumor growth suppression in the xenograft further supported the reverse effect of Met in primary resistant H1975 cells treated with Gef.

The fact that Met can inhibit growth of cancer cells is widely accepted, and the mechanism is associated with Met stimulating the activation of AMPK to inhibit mTOR phosphorylation $^{47,48}$ and that Met can reduce IGF-1R signaling and suppress the PI3K/AKT/mTOR axis. ${ }^{49,50}$ Some studies have shown that Met inhibits IGF-1R signaling in cancer cells. ${ }^{51-53}$ In addition, our group has reported that Met could restore crizotinib sensitivity in crizotinib-resistant NSCLC cells through inhibition of the IGF-1R signaling pathway. ${ }^{54}$ To further explore the mechanism involved in the cytotoxicity effect of Met, we investigated the IGF-1R signaling pathway. We found that Met treatment alone or in combination with Gef could suppress IGF-1R signaling and subsequently inactivate AKT protein. To confirm the importance of the inhibitory effect of Met on IGF-1R in H1975 cells, we knocked down IGF-1R by siRNA and determined cell proliferation. As a result, knockdown of IGF-1R diminished the sensitization role of Met in H1975 cells. Caveolin-1 is reported to be required for Met inhibitory effect on IGF1 action in NSCLC. ${ }^{55}$ In this context, whether caveolin-1 contributes to the synergistic inhibitory effect of Met on H1975 cells is deemed worthy of further study. Taken together, these results suggested the critical role of IGF-1R/AKT signaling in Met-regulated growth inhibition in H1975 cells, and Met-sensitized H1975 cells to Gef, via inhibition of mainly IGF-1R.

In the present study, we observed that after the H1975 cells were treated with Met alone, the protein level of BIM was increased and the combination of Met and Gef increased the BIM protein level to a greater extent. Consistent with our previous data, flow cytometry assay revealed that this 
A

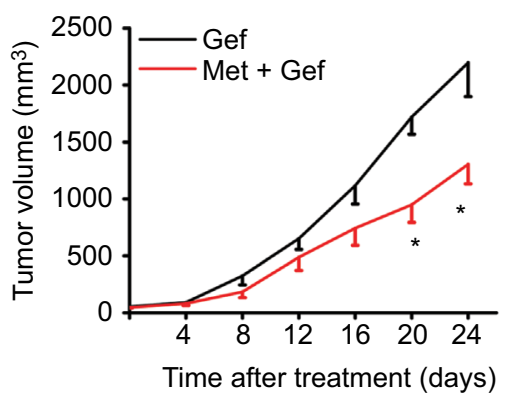

B

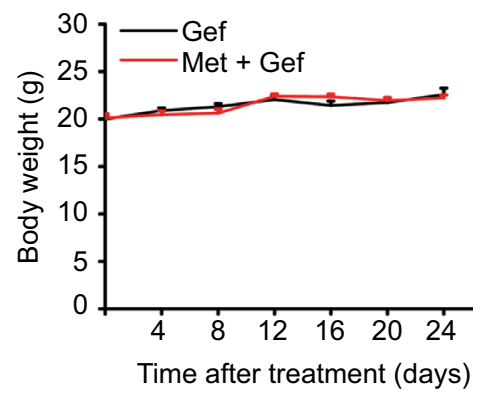

C

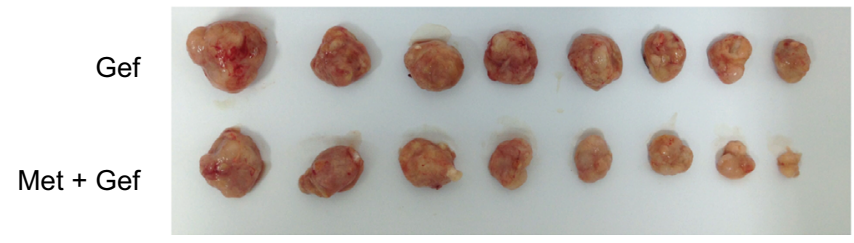

Figure 6 Addition of Met to Gef enhanced antitumor activity of EGFR-TKI in primary resistant tumor xenograft.

Notes: These nude mice were transplanted with HI 975 cells and assigned to two groups randomly after tumors were grown to appropriate volume, which were Gef (250 mg/L p.o.) and Met ( $\mathrm{l} \mathrm{mg/mL} \mathrm{p.o.),} \mathrm{for} 4$ weeks, each containing eight mice. The tumor area was measured every 4 days, and the tumor volume and body weight were calculated as described in the "Materials and methods" section. (A) Tumor volume $\left(\mathrm{mm}^{3}\right)$ of $\mathrm{H} 1975$ cells treated with Gef, and Met in combination with Gef; * $p<0.05$ when compared with the combinational group. (B) Body weight of the xenograft model after drug treatment. (C) Macroscopic appearance of the tumors at 4 weeks after drug administration. Abbreviations: Met, metformin; Gef, gefitinib, EGFR-TKI, epidermal growth factor receptor tyrosine kinase inhibitor; p.o., oral administration.

combination treatment effectively improved the apoptosis rate of H1975 cells. These results demonstrated that the antiproliferative synergistic effect of Met on H1975 cells with Gef was through inhibiting IGF-1R/AKT signaling, which consequently upregulated expression of BIM protein and promoted cell apoptosis through the activation of the mitochondrial pathway.

In addition, our laboratory has confirmed that for the sensitive cell lines PC-9 and HCC827, Gef alone could inhibit the p-EGFR activation; for the resistant cell lines PC-9GR and H1975, which harbor the T790M mutation, Gef showed poor performance, resulting in a high p-EGFR expression. Because Met treatment did inhibit the activation status of EGFR in NSCLC, ${ }^{56,57}$ it is possible that the role of Met in overcoming intrinsic resistance to EGFR-TKIs involves not only inhibiting IGF-1R signaling but also influencing the expression of $\mathrm{p}-\mathrm{EGFR}$.

\section{Conclusion}

Suppression of the IGF-1R pathway could restore EGFR-TKI primary resistant NSCLC H1975 cells' sensitivity to Gef, and synergistic antitumor effect was mediated through downregulation of p-AKT and escalation of BIM. We have reported the first evidence that Met inhibited IGF-1R signaling pathway and modulated AKT/BIM pathway, which contributed to the growth suppression and apoptosis induction role of Met, that potentiated the cytotoxicity effect of Gef in EGFR-TKI primary resistant H1975 cells. Moreover, the combination of Met and Gef showed greater inhibition of tumor growth than Gef alone in the xenograft. These findings provide a promising combined therapy strategy for the treatment of EGFR-TKI de novo resistant NSCLC.

\section{Acknowledgments}

This work was supported by the National Nature Science Foundation of China (81071912, 81472189). Yong-hong Pan is the first author to this work.

\section{Author contributions}

YHP carried out the flow cytometry analysis, small interfering RNA technology and Western blot analysis; performed the statistical analysis and drafted the manuscript. CYL and CHL carried out MTT, Ki67 incorporation assay and xenograft tumor experiment. LJ participated in the study design and coordination and helped to draft the manuscript. LL, HYC and YBW participated in the design of the study. YH conceived the study. All authors contributed toward data analysis, drafting and revising the paper and agree to be accountable for all aspects of the work. All authors read and approved the final manuscript. 


\section{Disclosure}

The authors report no conflicts of interest in this work.

\section{References}

1. Inoue A, Kobayashi K, Maemondo M, et al. Updated overall survival results from a randomized phase III trial comparing gefitinib with carboplatin-paclitaxel for chemo-naive non-small cell lung cancer with sensitive EGFR gene mutations (NEJ002). Ann Oncol. 2013;24(1):54-59.

2. Rosell R, Carcereny E, Gervais R, et al. Erlotinib versus standard chemotherapy as first-line treatment for European patients with advanced EGFR mutation-positive non-small-cell lung cancer (EURTAC): a multicentre, open-label, randomised phase 3 trial. Lancet Oncol. 2012;13(3):239-246.

3. Han JY, Park K, Kim SW, et al. First-SIGNAL: first-line single-agent iressa versus gemcitabine and cisplatin trial in never-smokers with adenocarcinoma of the lung. J Clin Oncol. 2012;30(10):1122-1128.

4. Mok T, Yang JJ, Lam KC. Treating patients with EGFR-sensitizing mutations: first line or second line - is there a difference? J Clin Oncol. 2013;31(8):1081-1088.

5. Jackman D, Pao W, Riely GJ, et al. Clinical definition of acquired resistance to epidermal growth factor receptor tyrosine kinase inhibitors in non-small-cell lung cancer. J Clin Oncol. 2010;28(2):357-360.

6. Lee JK, Shin JY, Kim S, et al. Primary resistance to epidermal growth factor receptor (EGFR) tyrosine kinase inhibitors (TKIs) in patients with non-small-cell lung cancer harboring TKI-sensitive EGFR mutations: an exploratory study. Ann Oncol. 2013;24(8):2080-2087.

7. Park KS, Raffeld M, Moon YW, et al. CRIPTO1 expression in EGFRmutant NSCLC elicits intrinsic EGFR-inhibitor resistance. J Clin Invest. 2014;124(7):3003-3015.

8. Mok TS, Wu YL, Thongprasert S, et al. Gefitinib or carboplatin-paclitaxel in pulmonary adenocarcinoma. N Engl J Med. 2009;361:947-957.

9. Maemondo M, Inoue A, Kobayashi K, et al. Gefitinib or chemotherapy for non-small-cell lung cancer with mutated EGFR. $N$ Engl J Med. 2010;362(25):2380-2388.

10. Mitsudomi T, Morita S, Yatabe Y, et al. Gefitinib versus cisplatin plus docetaxel in patients with non-small-cell lung cancer harbouring mutations of the epidermal growth factor receptor (WJTOG3405): an open label, randomised phase 3 trial. Lancet Oncol. 2010;11(2):121-128.

11. Zhou C, Wu YL, Chen G, et al. Erlotinib versus chemotherapy as first-line treatment for patients with advanced EGFR mutationpositive non-small cell lung cancer (OPTIMAL, CTONG-0802): a multicentre, open-label, randomised, phase 3 study. Lancet Oncol. 2011;12(8):735-742.

12. Cai H, Zhang Y, Han TK, Everett RS, Thakker DR. Cation-selective transporters are critical to the AMPK-mediated antiproliferative effects of metformin in human breast cancer cells. Int $J$ Cancer. 2016;138(9):2281-2292.

13. Yang J, Wei J, Wu Y, et al. Metformin induces ER stress-dependent apoptosis through $\mathrm{miR}-708-5 \mathrm{p} / \mathrm{NNAT}$ pathway in prostate cancer. Oncogenesis. 2015;4:e158.

14. Li B, Li X, Ni Z, et al. Dichloroacetate and metformin synergistically suppress the growth of ovarian cancer cells. Oncotarget. 2016;7(37):59458-59470.

15. Quinn BJ, Kitagawa H, Memmott RM, Gills JJ, Dennis PA. Repositioning metformin for cancer prevention and treatment. Trends Endocrinol Metab. 2013;24(9):469-480.

16. Chen H, Yao W, Chu Q, et al. Synergistic effects of metformin in combination with EGFR-TKI in the treatment of patients with advanced non-small cell lung cancer and type 2 diabetes. Cancer Lett. 2015;369(1):97-102.

17. Li L, Han R, Xiao H, et al. Metformin sensitizes EGFR-TKI-resistant human lung cancer cells in vitro and in vivo through inhibition of il-6 signaling and EMT reversal. Clin Cancer Res. 2014;20(10):2714-2726.
18. Sequist LV, Waltman BA, Dias-Santagata D, et al. Genotypic and histological evolution of lung cancers acquiring resistance to EGFR inhibitors. Sci Transl Med. 2011;3(75):75ra26.

19. Arcila ME, Oxnard GR, Nafa K, et al. Rebiopsy of lung cancer patients with acquired resistance to EGFR inhibitors and enhanced detection of the T790M mutation using a locked nucleic acid-based assay. Clin Cancer Res. 2011;17(5):1169-1180.

20. Yu HA, Arcila ME, Rekhtman N, et al. Analysis of tumor specimens at the time of acquired resistance to EGFR-TKI therapy in 155 patients with EGFR-mutant lung cancers. Clin Cancer Res. 2013;19(8):2240-2247.

21. $\mathrm{Hu} \mathrm{S}, \mathrm{Fu} \mathrm{W}, \mathrm{Li}$ T, et al. Antagonism of EGFR and Notch limits resistance to EGFR inhibitors and radiation by decreasing tumor-initiating cell frequency. Sci Transl Med. 2017;9(380):eaag0339.

22. Zanella ER, Galimi F, Sassi F, et al. IGF2 is an actionable target that identifies a distinct subpopulation of colorectal cancer patients with marginal response to anti-EGFR therapies. Sci Transl Med. 2015;7(272): $272 \mathrm{ra} 12$.

23. Cappuzzo F, Janne PA, Skokan M, et al. MET increased gene copy number and primary resistance to gefitinib therapy in non-small-cell lung cancer patients. Ann Oncol. 2009;20(2):298-304.

24. Tanaka A, Sueoka-Aragane N, Nakamura T, et al. Co-existence of positive MET FISH status with EGFR mutations signifies poor prognosis in lung adenocarcinoma patients. Lung Cancer. 2012;75(1):89-94.

25. Ng KP, Hillmer AM, Chuah CT, et al. A common BIM deletion polymorphism mediates intrinsic resistance and inferior responses to tyrosine kinase inhibitors in cancer. Nat Med. 2012;18(4):521-528.

26. Sos ML, Koker M, Weir BA, et al. PTEN loss contributes to erlotinib resistance in EGFR-mutant lung cancer by activation of Akt and EGFR. Cancer Res. 2009;69:3256-3261.

27. Inukai $\mathrm{M}$, Toyooka $\mathrm{S}$, Ito $\mathrm{S}$, et al. Presence of epidermal growth factor receptor gene T790M mutation as a minor clone in non-small cell lung cancer. Cancer Res. 2006;66(16):7854-7858.

28. Su KY, Chen HY, Li KC, et al. Pretreatment epidermal growth factor receptor (EGFR) T790M mutation predicts shorter EGFR tyrosine kinase inhibitor response duration in patients with non-small-cell lung cancer. J Clin Oncol. 2012;30(4):433-440.

29. Dong S, Qu X, Li W, et al. The long non-coding RNA, GAS5, enhances gefitinib-induced cell death in innate EGFR tyrosine kinase inhibitorresistant lung adenocarcinoma cells with wide-type EGFR via downregulation of the IGF-1R expression. J Hematol Oncol. 2015;8:43.

30. Masago K, Fujita S, Togashi Y, et al. Clinical significance of epidermal growth factor receptor mutations and insulin-like growth factor 1 and its binding protein 3 in advanced non-squamous non-small cell lung cancer. Oncol Rep. 2011;26(4):795-803.

31. Kim GW, Song JS, Choi CM, et al. Multiple resistant factors in lung cancer with primary resistance to EGFR-TK inhibitors confer poor survival. Lung Cancer. 2015;88(2):139-146.

32. Ichite N, Chougule MB, Jackson T, Fulzele SV, Safe S, Singh M. Enhancement of docetaxel anticancer activity by a novel diindolylmethane compound in human non-small cell lung cancer. Clin Cancer Res. 2009;15(2):543-552.

33. Morgillo F, Woo JK, Kim ES, Hong WK, Lee HY. Heterodimerization of insulin-like growth factor receptor/epidermal growth factor receptor and induction of survivin expression counteract the antitumor action of erlotinib. Cancer Res. 2006;66(20):10100-10111.

34. Morgillo F, Kim WY, Kim ES, Ciardiello F, Hong WK, Lee HY. Implication of the insulin-like growth factor-IR pathway in the resistance of non-small cell lung cancer cells to treatment with gefitinib. Clin Cancer Res. 2007;13(9):2795-2803.

35. Guix M, Faber AC, Wang SE, et al. Acquired resistance to EGFR tyrosine kinase inhibitors in cancer cells is mediated by loss of IGF-binding proteins. J Clin Invest. 2008;118(7):2609-2619.

36. Cortot AB, Repellin CE, Shimamura T, et al. Resistance to irreversible EGF receptor tyrosine kinase inhibitors through a multistep mechanism involving the IGF1R pathway. Cancer Res. 2013;73(2):834-843. 
37. Choi YJ, Rho JK, Jeon BS, et al. Combined inhibition of IGFR enhances the effects of gefitinib in H1650: a lung cancer cell line with EGFR mutation and primary resistance to EGFR-TK inhibitors. Cancer Chemother Pharmacol. 2010;66(2):381-388.

38. Sheppard K, Kinross KM, Solomon B, Pearson RB, Phillips WA. Targeting PI3 kinase/AKT/mTOR signaling in cancer. Crit Rev Oncog. 2012;17(1):69-95.

39. Vázquez de la Torre A, Junyent F, Folch J, et al. PI3k/akt inhibition induces apoptosis through p38 activation in neurons. Pharmacol Res. 2013;70(1):116-125.

40. Lim SM, Kim HR, Cho EK, et al. Targeted sequencing identifies genetic alterations that confer primary resistance to EGFR tyrosine kinase inhibitor (Korean Lung Cancer Consortium). Oncotarget. 2016;7(24):36311-36320.

41. Gallagher EJ, LeRoith D. The proliferating role of insulin and insulin-like growth factors in cancer. Trends Endocrinol Metab. 2010;21(10):610-618.

42. van der Veeken J, Oliveira S, Schiffelers RM, Storm G, van Bergen En Henegouwen PM, Roovers RC. Crosstalk between epidermal growth factor receptor- and insulin-like growth factor-1 receptor signaling: implications for cancer therapy. Curr Cancer Drug Targets. 2009;9(6):748-760.

43. Prenek L, Boldizsár F, Kugyelka R, et al. The regulation of the mitochondrial apoptotic pathway by glucocorticoid receptor in collaboration with Bcl-2 family proteins in developing T cells. Apoptosis. 2017;22(2):239-253.

44. Ishii Y, Nhiayi MK, Tse E, et al. Knockout serum replacement promotes cell survival by preventing BIM from inducing mitochondrial cytochrome C release. PLoS One. 2015;10(10):e0140585.

45. Rosich L, Saborit-Villarroya I, López-Guerra M, et al. The phosphatidylinositol-3-kinase inhibitor NVP-BKM120 overcomes resistance signals derived from microenvironment by regulating the Akt/FoxO3a/ Bim axis in chronic lymphocytic leukemia cells. Haematologica. 2013;98(11):1739-1747.

46. Aroui S, Dardevet L, Najlaoui F, et al. PTEN-regulated AKT/FoxO3a/ Bim signaling contributes to human cell glioblastoma apoptosis by platinum-maurocalcin conjugate. Int J Biochem Cell Biol. 2016;77(pt A):15-22.
47. Luo Z, Zang M, Guo W. AMPK as a metabolic tumor suppressor: control of metabolism and cell growth. Future Oncol. 2010;6(3):457-470.

48. Xu JN, Zeng $\mathrm{C}$, Zhou Y, et al. Metformin inhibits StAR expression in human endometriotic stromal cells via AMPK mediated disruption of CREB-CRTC2 complex formation. J Clin Endocrinol Metab. 2014;99(8):2795-2803.

49. Vazquez-Martin A, Oliveras-Ferraros C, Del Barco S, et al. If mammalian target of metformin indirectly is mammalian target of rapamycin, then the insulin-like growth factor-1 receptor axis will audit the efficacy of metformin in cancer clinical trials. J Clin Oncol. 2009;27(33):e207-e209.

50. Martin-Castillo B, Vazquez-Martin A, Oliveras-Ferraros C, Menendez JA. Metformin and cancer: doses, mechanisms and the dandelion and hormetic phenomena. Cell Cycle. 2010;9(6):1057-1064.

51. Abo-Elmatty DM, Ahmed EA, Tawfik MK, Helmy SA. Metformin enhancing the antitumor efficacy of carboplatin against Ehrlich solid carcinoma grown in diabetic mice: effect on IGF-1 and tumoral expression of IGF-1 receptors. Int Immunopharmacol. 2017;44:72-86.

52. Tandon M, Chen Z, Othman AH, Pratap J. Role of Runx 2 in IGF-1Rß/Aktand AMPK/Erk-dependent growth, survival and sensitivity towards metformin in breast cancer bone metastasis. Oncogene. 2016;35(36):4730-4740.

53. Karnevi E, Said K, Andersson R, Rosendahl AH. Metformin-mediated growth inhibition involves suppression of the IGF-I receptor signalling pathway in human pancreatic cancer cells. BMC Cancer. 2013;13:235.

54. Li L, Wang Y, Peng T, et al. Metformin restores crizotinib sensitivity in crizotinib-resistant human lung cancer cells through inhibition of IGF1-R signaling pathway. Oncotarget. 2016;7(23):34442-34452.

55. Salani B, Maffioli S, Hamoudane M, et al. Caveolin-1 is essential for metformin inhibitory effect on IGF1 action in non-small-cell lung cancer cells. FASEB J. 2012;26(2):788-798.

56. Xiao Z, Sperl B, Ullrich A, Knyazev P. Metformin and salinomycin as the best combination for the eradication of NSCLC monolayer cells and their alveospheres (cancer stem cells) irrespective of EGFR, KRAS, EML4/ALK and LKB1 status. Oncotarget. 2014;5(24):12877-12890.

57. Morgillo F, Sasso FC, Della Corte CM, et al. Synergistic effects of metformin treatment in combination with gefitinib, a selective EGFR tyrosine kinase inhibitor, in LKB1 wild-type NSCLC cell lines. Clin Cancer Res. 2013;19(13):3508-3519.
Biologics: Targets and Therapy

\section{Publish your work in this journal}

Biologics: Targets and Therapy is an international, peer-reviewed journal focusing on the patho-physiological rationale for and clinical application of Biologic agents in the management of autoimmune diseases, cancers or other pathologies where a molecular target can be identified. This journal is indexed on PubMed Central, EMBase, and Scopus.

\section{Dovepress}

The manuscript management system is completely online and includes a very quick and fair peer-review system, which is all easy to use Visit http://www.dovepress.com/testimonials.php to read real quotes from published authors. 